\title{
Foxg1 deletion impairs the development of the epithalamus
}

\author{
Bin Liu', Kaixing Zhou', Xiaojing Wu' and Chunjie Zhao ${ }^{1,2^{*}}$
}

\begin{abstract}
The epithalamus, which is dorsal to the thalamus, consists of the habenula, pineal gland and third ventricle choroid plexus and plays important roles in the stress response and sleep-wake cycle in vertebrates. During development, the epithalamus arises from the most dorsal part of prosomere 2. However, the mechanism underlying epithalamic development remains largely unknown. Foxg1 is critical for the development of the telencephalon, but its role in diencephalic development has been under-investigated. Patients suffering from FOXG1-related disorders exhibit severe anxiety, sleep disturbance and choroid plexus cysts, indicating that Foxg1 likely plays a role in epithalamic development. In this study, we identified the specific expression of Foxg1 in the developing epithalamus. Using a "self-deletion" approach, we found that the habenula significantly expanded and included an increased number of habenular subtype neurons. The innervations, particularly the habenular commissure, were severely impaired. Meanwhile, the Foxg1 mutants exhibited a reduced pineal gland and more branched choroid plexus. After ablation of Foxg1 no obvious changes in Shh and Fgf signalling were observed, suggesting that Foxg1 regulates the development of the epithalamus without the involvement of Shh and Fgfs. Our findings provide new insights into the regulation of the development of the epithalamus.
\end{abstract}

Keywords: Epithalamus, Habenula, Pineal gland, Choroid plexus, FOXG1-related disorders, Sleep disturbance, Fgf15

\section{Introduction}

The epithalamus, which consists of the habenula, pineal gland, and third ventricle choroid plexus $\left(3^{\text {rd }} \mathrm{Chp}\right)$, is involved in many functions, including motor control, the sleep-wake cycle and stress responses [1-3]. The habenula is highly conserved in vertebrates and acts as a critical node connecting the forebrain to the midbrain and hindbrain by receiving inputs from the limbic system and the basal ganglia and projecting to the monoaminergic nuclei $[4,5]$. The pineal gland is critical for the regulation of circadian rhythms due to its production of melatonin [6], and the choroid plexus synthesizes cerebrospinal fluid (CSF) and many growth factors, including fibroblasts and insulin-like and platelet-derived growth factors, and plays important roles, such as providing a route for nutrients and removing by-products of metabolism [7, 8]. Dysfunction of the epithalamus has been reported to be related to mood disorders, such as

\footnotetext{
* Correspondence: zhaocj@seu.edu.cn

${ }^{1}$ Key Laboratory of Developmental Genes and Human Diseases, MOE, School of Medicine, Southeast University, Nanjing 210009, People's Republic of China

${ }^{2}$ Depression Center, Institute for Brain Disorders, Beijing 100069, China
}

major depression, and schizophrenia and sleeping disorders $[2,9-12]$. However, knowledge regarding the developmental process of the epithalamus is limited.

During early development, the progenitor domain in the diencephalon is divided into three prosomeres (p), i.e., p1, $\mathrm{p} 2$, and $\mathrm{p} 3$, along the anterior-posterior axis $[13,14]$. P1 and $\mathrm{p} 3$ give rise to the pretectum and prethalamus, respectively. The most dorsal region of $\mathrm{p} 2$ produces the epithalamus, and the other part generates the thalamus. In the presumptive epithalamic progenitor domain, the most anterior area containing the roof plate develops into the $3{ }^{\text {rd }} \mathrm{Chp}$, while the adjacent part generates the habenular commissure, paired habenulas and pineal gland. Previously, a member of the fibroblast growth factor (Fgf) family, $F g f 8$, has been reported to regulate the development of the habenula and pineal gland in a dosedependent manner [15]. In zebrafish, Fgf signalling also controls the specification of the pineal complex [16]. However, the molecular and cellular mechanisms underlying the development of the epithalamus still remain largely unknown. 
Foxg1 encodes a winged-helix transcriptional repressor and has been reported to play critical roles during telencephalic development [17-20]. Patients with mutations in FOXG1 have been reported to suffer from mental retardation, poor social interactions and severe anxiety [21]. Notably, severe sleep disturbance, deformation of the third ventricle and choroid plexus cysts have also been reported [22, 23]. Thus, Foxg1 may also be involved in the regulation of epithalamic development. In the present study, we found that a disruption of Foxg1 leads to an impaired epithalamus with an expanded habenula, a smaller pineal gland and an extremely complicated choroid plexus. Various subtypes of neurons in the habenula exhibited a remarkable increase in number with impaired innervations. Furthermore, ablation of Foxg1 led to the abnormal sub-regionalization of the epithalamic progenitor domain. Our data provide novel perspectives regarding the development of the epithalamus.

\section{Methods}

\section{Animals}

Foxg1-Cre (Foxg1 tm1(cre)Skm ) [24] mouse line was purchased from the Jackson laboratory (US, Foxg1-Cre stock: 006084). The Foxg $1^{f l f l}$ line was obtained as previously described [19, 25]. The Fzd10-EGFP transgenic line was generated using standard methods (unpublished data). Foxg1 disruption was achieved by an intercross of Foxg1-cre or crossing Foxg1-cre with Foxg $1^{f l+t}$. Both Foxg1 ${ }^{\text {cre-cre }}$ and Foxg1-cre;Foxg1 $1^{f /+}$ were considered mutants, and their wild-type littermates and Foxg $f^{f /+}$ were considered controls. All animals were maintained on an outbred CD1 genetic background and were housed in the animal facility of the Southeast University. All experimental procedures followed the guidelines approved by Southeast University. To stage the embryos, the mice were mated in the afternoon. The day the vaginal plug was found at noon was considered embryonic day 0.5 (E0.5), and the day of birth was considered postnatal day 0 (P0).

\section{Tissue processing and Nissl staining}

Embryonic brains were directly dissected in cold phosphate buffered saline (PBS) and immediately transferred to $4 \%$ paraformaldehyde (PFA, Sigma-Aldrich, 441,244, US) overnight at $4{ }^{\circ} \mathrm{C}$. The brains from P0 were perfused and then post-fixed at $4{ }^{\circ} \mathrm{C}$ for $12-16 \mathrm{~h}$. The brains were then cryoprotected in 30\% sucrose and embedded in OCT. Coronal sections $(8-12 \mu \mathrm{m}$ thick) were obtained using a Leica cryostat $(\mathrm{CM} 3050 \mathrm{~S})$ and stored at $-70{ }^{\circ} \mathrm{C}$ until use. The Nissl staining was performed according to standard protocols.

\section{In situ hybridization}

E12.5 brains were dissected, immediately transferred to $500 \mu \mathrm{L}$ of TRI Reagent (Sigma-Aldrich, T9424, US) and processed for total RNA isolation according to the manufacturer's instructions. After purification using the RNeasyPlus Mini Kit (QIAGEN, 74,106, DE), the RNA concentration was measured using an Agilent 2100 Bioanalyser (Agilent Technologies, Palo Alto, CA). In total, $2 \mu \mathrm{g}$ of purified total RNA was used as the template to synthesize cDNA using the PrimeScript ${ }^{\mathrm{T}} \mathrm{RT}$ Master Mix (Takara, RR036A, CN). The cDNA was then used as the template to amplify DNA fragments by PCR for the probe synthesis. The PCR products were inserted into the pBlueScript vector by $\mathrm{T} 4$ ligation polymerase (Takara, 2040A, CN). The probes were synthesized using the Digoxigenin-labelling Mix (Roche, 11,277,073,910, DE) and T3 RNA polymerase (Roche, 11,031,171,001, DE) or T7 RNA polymerase (Roche, 10,881,175,001, DE). The in situ hybridization was performed as previously described [26, 27].

\section{Immunofluorescence}

Immunofluorescence was performed as previously described [19]. The primary antibodies and dilutions were as follows: anti-Calretinin (Millipore, AB5054, 1:500); anti-Calbindin (Millipore, AB1778, 1:250); anti-Foxg1 (Abcam, ab18259, 1:1000); anti-GFP (Abcam, ab13970, 1:1000); anti-L1 (Millipore, MAB5272, 1:500); anti-Pax6 (BioLegend, 901,301, 1:1000); and anti-Vglut2 (Millipore, MAB5504, 1:500). The secondary antibodies used were Alexa Fluro 488 donkey anti-chicken (Jackson Lab, 703-545-155, 1:500), Alexa Fluor 488 donkey antirabbit (Life, A21206, 1:500), Alexa Fluor 546 donkey anti-rabbit (Life, A10040, 1:500), Alexa Fluro 647 donkey anti-rabbit (Life, A31573, 1:500), Alexa Fluor 488 donkey anti-rat (Life, A21208, 1:500), CF 633 donkey anti-rat (Sigma-Aldrich, SAB4600133, 1:500) and Alexa Fluro 647 donkey anti-mouse (Invitrogen, A21236, 1:500).

\section{Statistical analysis and cell counting}

The measurements for the volumetric analyses were performed using every tenth $8 \mu \mathrm{m}$ coronal section stained with anti-Calretinin. The regions of the habenula were measured using ImageJ software as previously described $[28,29]$. The volumes $(\mathrm{V})$ were calculated as $\mathrm{V}=\sum \mathrm{A} \times \mathrm{i} \times \mathrm{d}$, according to Cavalieri's principle, where A represents the sum of the areas in the habenula, I represents the intervals between the sections, and $d$ represents the thickness of the sections. The measurements for analysis of the thickness of habenular commissure were performed using every third $10 \mu \mathrm{m}$ coronal section by the immunofluorescence of L1. The thickness at the midline area were measured by ImageJ software and calculated by the average. Cells of each distinct cell type in the sub-nuclei of the habenula were counted, and the numbers of $\mathrm{CR}^{+}, \mathrm{CB}^{+}, \mathrm{Tacl}^{+}$and $\mathrm{Pax}^{+}$ cells were counted in every tenth $8 \mu \mathrm{m}$ coronal section from each side of the habenula and summed to obtain the 
total number. We considered every strong $\mathrm{Tac}^{+}{ }^{+}$staining dot as a single cell under high magnification views. Very weak staining was not taken into account. Both controls and mutants were counted under the same criterion. The

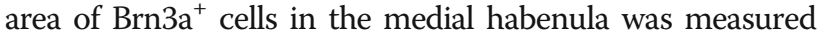
by ImageJ software in every third $10 \mu \mathrm{m}$ coronal section from one side of the habenula and averaged to obtain the mean area per section. All experiments were performed using at least three different litters, and the data were statistically analysed using GraphPad Prism software. Two-tailed Student's t-test was performed to analyse the statistical significance at $p<0.05{ }^{(*)}, p<0.011^{(* *)}$ and $p<0.0011^{(* * * *)}$.

\section{Quantitative real time polymerase chain reaction (qRT-PCR)}

qRT-PCR was carried out according to the protocols as previously described [19]. The dorsal part of E12.5 diencephalon at least from three different litters were used. The specific primers for $F g f 15$ is: $5^{\prime}$-GAGGAAGC CAGAAGGTATGAAG-3' and ' 5'-GGCAAGCTAAGA TCCCATGA- 3'.

\section{Results \\ Foxg 1 is specifically expressed in the developing dorsal diencephalon, and ablation of Foxg 1 leads to an impaired epithalamus}

The forkhead box transcription factor Foxg1 has been reported to be critical for telencephalic development [17]. However, the function of Foxg1 in the diencephalon has been under-investigated. Considering the symptoms, including poor sleep patterns, emotional disorders and choroid plexus cysts, observed in patients suffering from FOXG1 syndrome [21-23], we suspect that Foxg1 plays an important role in the developing diencephalon. Previously, the forced overexpression of Foxg1 in chicks has been shown to downregulate Otx2 in the alar plate of the diencephalon, indicating that Foxg1 likely plays a role in diencephalic development [30]. In this study, we first analysed the expression of Foxg1 in detail using in situ hybridization. As shown in Fig. 1a, at E12.5, although extremely strong staining was detected in the developing telencephalon, Foxg1 was also found to be weakly expressed in the progenitors in the third ventricular zone $\left(3^{\text {rd }} \mathrm{VZ}\right)$ and their postmitotic derivatives. This expression pattern was confirmed by immunostaining with anti-Foxg1 (Fig. 1b, arrow). Strong staining was particularly detected at the dorsal-most region of the diencephalon, which was presumably the epithalamus area from which the habenula, pineal gland and $3{ }^{\text {rd }} \mathrm{Chp}$ arise (Fig. 1a, b). As development proceeded, the expression level of Foxg1 gradually increased with stronger expression in the medial habenula $(\mathrm{MHb})$ and weaker expression in the lateral habenula (LHb) at E18.5 (Fig. 1d, e). Previously, a new Foxg1-IRES-Cre line that faithfully recapitulates the endogenous Foxg1 expression also exhibited Cre-medicated recombination in the developing epithalamus [31], which is consistent with our observations. Collectively, Foxg1 is specifically expressed in the developing diencephalon, particularly in the dorsal part of the $3^{\text {rd }} \mathrm{VZ}$, strongly supporting that Foxg1 plays a role in the development of the epithalamus.

Subsequently, we adopted the "self-deletion" approach by crossing Foxg1-Cre with Foxg1 $1^{f l / f l}$ to obtain compound homozygous Foxg1 $1^{\text {crelfl }}$ mice [32]. Both Foxg1 $1^{\text {cre/fl }}$ and Foxg1 $1^{\text {cre/cre }}$ were considered Foxg1-phenotypical null mutants and in this study, all results were obtained in comparable levels from serial sections of the habenula. As shown in Fig. 1c and f, the expression of Foxg1 in the dorsal diencephalon were effectively eliminated in the mutants at E12.5 and E18.5. We first analysed the changes of the epithalamus during early development. Previously, Brn3a (also called Pou4f1) has been reported to be strongly expressed in postmitotic neurons in the $\mathrm{MHb}$ and weakly expressed in the LHb and critical for the development of the habenula [33]. At the stages of E12.5 and E14.5, in situ staining of Brn3a showed the habenula was slightly enlarged and expanded dorsal-laterally after Foxg1 deletion (Fig. 1g, g'; h, h'). This is confirmed by Nissl staining as well (Fig. 1i, i'). At E18.5, the habenula visualized by Nissl staining expanded much more than that of observed in E12.5 and E14.5. The third ventricle was found to be significantly enlarged which could be a result of the changes caused in the lateral ventricle due to the decrease in the size of the cortex as previously reported [34, 35] (Fig. 1j, j'). However, the pineal gland was smaller (Fig. 1k, k'). Interestingly, the $3^{\text {rd }} \mathrm{Chp}$ were more branched in Foxg1 mutants compared to the controls (Fig. 1k, k', asterisk). To further confirm these abnormalities, we performed in situ hybridization for Frizzled10 (Fzd10), one of the Wnt receptors, specifically expressed in the $\mathrm{MHb}$ progenitors and postmitotic neurons [36]. As shown in Fig. 11-1', Fzd10 ${ }^{+}$region was enlarged. We also generated a Fzd10-EGFP transgenic mouse line in which EGFP faithfully reflected the endogenous Fzd10 expression (unpublished data). As shown in Fig. 1m-m', the $\mathrm{MHb}$ appeared to expand to the lateral side, resulting in an irregular shape, and the habenular commissure was lengthened and became thinner. A smaller pineal gland was also observed, which is consistent with the observations using Nissl staining. Finally, we evaluated the volume of the habenula and found that it was relative increased by approximately $12 \%$ in mutants (Fig. $1 \mathrm{n}$ ). Collectively, the disruption of Foxg1 caused an impaired epithalamus.

\section{Increased numbers of epithalamic progenitors and habenular subtype neurons}

To further investigate the cause of the enlarged habenula following the Foxg1 deletion, we examined the progenitor 


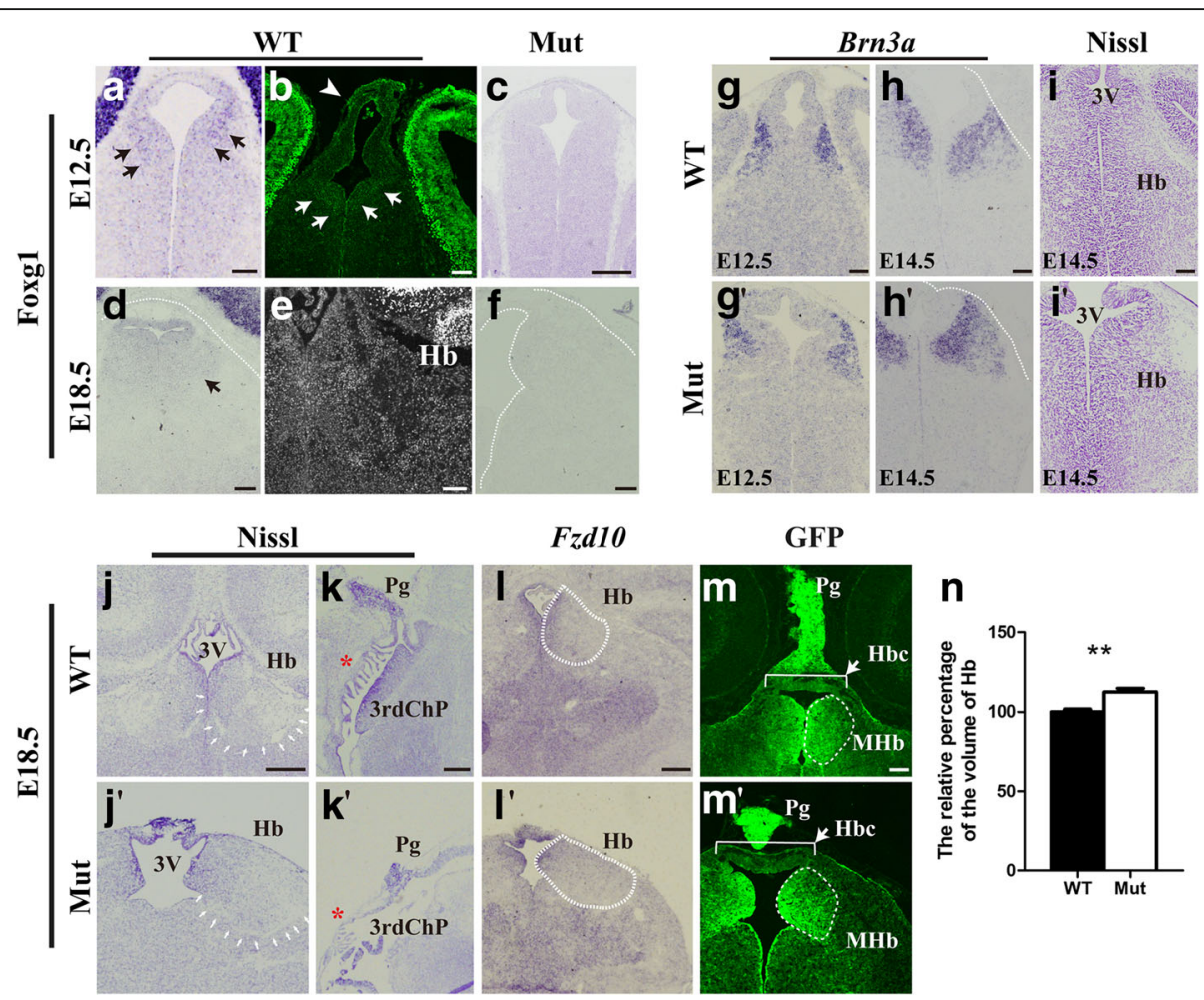

Fig. 1 Foxg 1 is required for the development of the epithalamus. (a and $\mathbf{b}$ ): In situ hybridization of Foxg 1 (a) and immunofluorescence of Foxg1 (b) in E12.5 coronal sections. Foxg1 is expressed at the dorsal developing diencephalic ventricular zone, particularly at the prospective habenular ventricular zone (arrows) and the pineal gland (arrowhead). (c): Foxg1 is effectively eliminated in Foxg1 mutants at E12.5. (d and e): In situ hybridization of Foxg1 (d) and immunofluorescence of Foxg1 (e) in E18.5 coronal sections. Arrow in d show the expression of Foxg1 in the habenula. (f): Foxg1 is effectively ablated at E18.5. (g-h'): In situ hybridization of E12.5 (g-g') and E14.5 (h-h') forebrain with Brn3a showing the habenula were slightly enlarged. (i, i'): Nissl staining of E14.5 forebrain in coronal sections revealed the enlarged habenula. The white dashed line in $\mathbf{d}, \mathbf{f}, \mathbf{h}$ and $\mathbf{h}^{\prime}$ outlined the diencephalon. ( $\left.\mathbf{j}-k^{\prime}\right)$ : Nissl staining in E18.5 coronal $\left(\mathbf{j}-\mathbf{j}^{\prime}\right)$ and sagittal sections ( $\left.\mathbf{k}-\mathrm{k}^{\prime}\right)$ showing the dorsolaterally expanded habenula $\left(\mathbf{j}, \mathbf{j}^{\prime}\right.$, arrows), enlarged third ventricle, more branched third ventricle choroid plexus ( $\mathbf{k}, \mathbf{k}^{\prime}$, asterisk) and the aberrant shape of the pineal gland in mutants. (I, I'): In situ hybridization revealed the Fzd10 ${ }^{+}$habenular region were enlarged. ( $\left.\mathbf{m}, \mathrm{m}^{\prime}\right)$ : Immunofluorescence with GFP showing the expanded habenula in the mutants $\left(\mathrm{m}^{\prime}\right)$ compared to that in the controls $(\mathbf{m})$, the elongated habenular commissure (bracket)

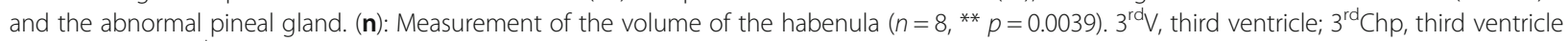
choroid plexus; $3^{\text {rd }} \mathrm{VZ}$, ventricular zone of the third ventricle; $\mathrm{Hb}$, habenula; $\mathrm{Hbc}$, habenular commissure; MHb, medial habenula; Pg, pineal gland. Scale bars: $100 \mu \mathrm{m}$

pool at E18.5. In the controls, Pax6 was expressed in the dorsal $3{ }^{\text {rd }} \mathrm{VZ}$, and its expression was particularly intense in the epithalamic progenitors. A portion of the $\mathrm{Pax}^{+}$ progenitor cells was also dispersed within the dorsal $\mathrm{MHb}$ (dMHb) (Fig. 2a, dotted line) and the lateral division of the LHb (LHbL) (Fig. 2a, yellow arrowhead). In the mutants, the epithalamic VZ seemed thicker than that in the controls, and more $\mathrm{Pax}^{+}$cells were scattered in the $\mathrm{dMHb}$ and LHbL; the total number of $\mathrm{Pax6}^{+}$cells was significantly increased by approximately $37 \%$ (Fig. 2a, a', i), demonstrating that disruption of Foxg1 results in an increased number of progenitors in the developing epithalamus. Then, we examined the alterations in several subtypes of habenular neurons. Calretinin $(C R)$ and Calbindin $(C B)$ are two members of the EF-hand family of calcium-binding proteins that are required for the differentiation of early generated thalamic neurons [37]. Here, we found that $\mathrm{CR}^{+}$neurons were mainly populated in the $\mathrm{dMHb}$ and $\mathrm{LHbL}$, which are the similar regions in which the $\mathrm{Pax}^{+}$progenitors were located; additionally, the $\mathrm{CR}^{+}$ neurons were distributed in the central part of the medial division of the $\mathrm{LHb}$ (LHbMC). The total number of $\mathrm{CR}^{+}$ cells in the habenula was remarkably increased by approximately $33 \%$, a $42 \%$ increase in the $\mathrm{dMHb}$ and a $22 \%$ increase in the LHbL were observed (Fig. $\left.2 b, b^{\prime} ; j\right)$. The CB ${ }^{+}$neurons were mainly detected in the boundary between the $\mathrm{MHb}$ and the LHb. In the mutants, the number of $\mathrm{CB}$ ${ }^{+}$neurons was also significantly increased by approximately $78 \%$, and more $\mathrm{CB}^{+}$neurons were scattered in the $\mathrm{LHb}$ (Fig. 2c, c'; k). In situ staining showed $\mathrm{Brn}^{\prime} \mathrm{a}^{+}$areas in both the mutant $\mathrm{MHb}$ and $\mathrm{LHb}$ were expanded (Fig. $2 \mathrm{~d}$, $\left.\mathrm{d}^{\prime}\right)$. We measured the Brn3a $\mathrm{a}^{+}$area at the central level of the $\mathrm{MHb}$ and found there was approximate $51 \%$ increase in mutants (Fig. 2l). 

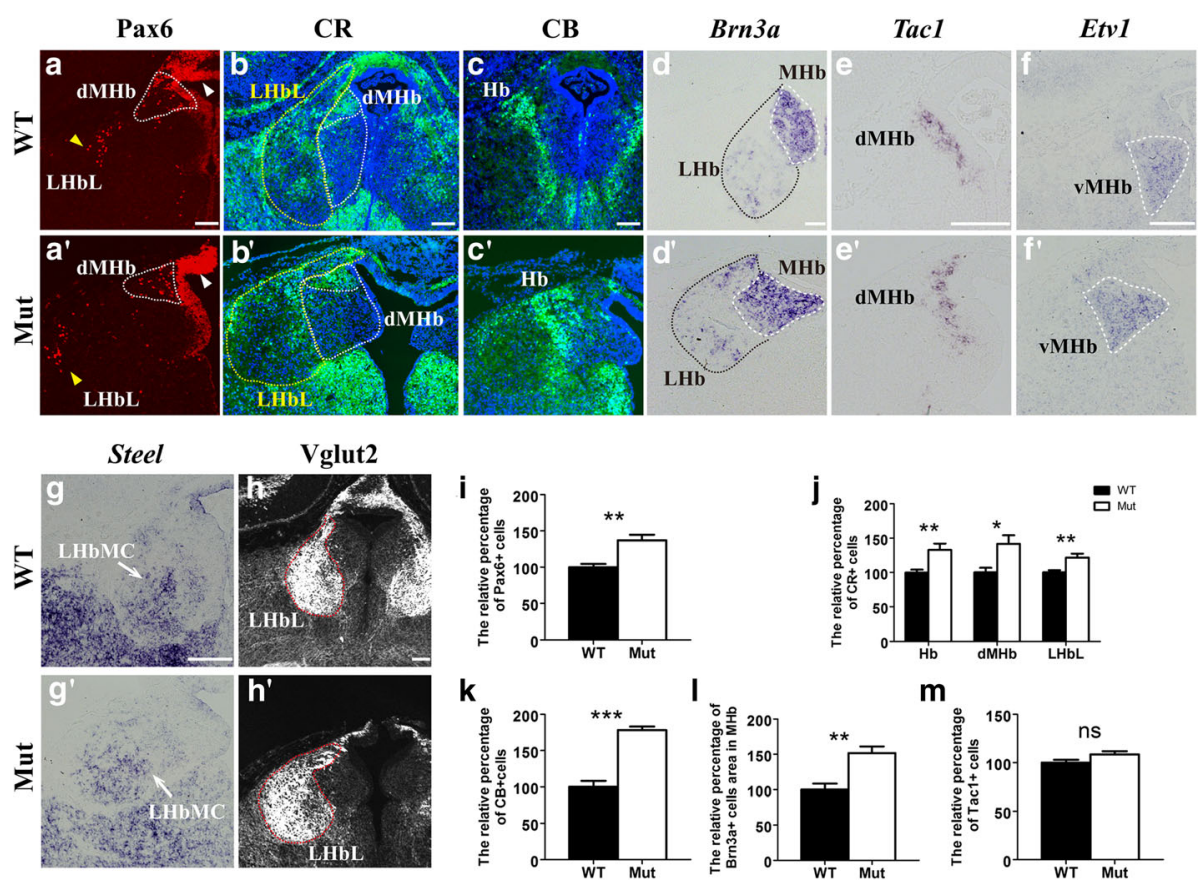

Fig. 2 Abnormal development of the habenular subtype neurons. (a-a'): Immunofluorescence of anti-Pax6. Pax $6^{+}$progenitors in the epithalamic VZ (white arrowhead) were remarkably increased compared to those in the controls, and more Pax $6^{+}$cells were scattered in the $\mathrm{dMHb}$ (dotted line) and LHbL (yellow arrowhead). (b-b'): Increased number of $\mathrm{CR}^{+}$subtype neurons in the $\mathrm{dMHb}$ (white dotted line) and LHbL (yellow dotted line). (c, $\mathbf{c}^{\prime}$ ): Increased numbers of $\mathrm{CB}^{+}$subtype neurons in the habenula. $\left(\mathbf{d}, \mathbf{d}^{\prime}\right)$ : In situ hybridization showing Brn3a strongly expressed in postmitotic neurons in the $\mathrm{MHb}$ (white dotted line) and weakly expressed in the LHb (black dotted line), and both stained areas were expanded with irregular morphologies in the Foxg1 mutants. (e-f'): Substance P-ergic neurons in the dMHb showing Tacl in situ hybridization and Etv $1^{+}$glutamatergic neurons in the vMHb. ( $\left.\mathbf{g}-h^{\prime}\right)$ : Enlarged LHbMC and LHbL. (i-m): Quantitative analysis of the numbers of Pax6 ${ }^{+}$progenitors $\left(\mathbf{i}, n=6,{ }^{* *} p=0.002\right), C R^{+}$neurons $(\mathbf{j}, n=6, H b$, $\left.{ }^{* *} p=0.0085 ; \mathrm{dMHb},{ }^{*} p=0.0148 ; \mathrm{LHbL},{ }^{* *} p=0.0069\right), \mathrm{CB}^{+}$neurons $\left(\mathbf{k}, \mathrm{n}=6,{ }^{* * *} p<0.0001\right)$, and Tac1 ${ }^{+}$neurons $(\mathbf{m}, \mathrm{n}=8, p=0.067)$. (I) Quantitative analysis of Brn3a ${ }^{+}$area in coronal section crossing the midmost level of the habenula $\left(\mathbf{I}, n=4,{ }^{* *} p=0.0063\right)$. The data are presented as the mean \pm S.E.M. Hb, habenula; $\mathrm{MHb}$, medial habenula; $\mathrm{dMHb}$, dorsal medial habenula; $\mathrm{vMHb}$, ventral medial habenula; $\mathrm{LHb}$, lateral habenula; LHbL, lateral division of the lateral habenula; LHbMC, central part of the medial division of the lateral habenula. Scale bars: $100 \mu \mathrm{m}$

We further analysed the changes in several other neuronal subtypes in the MHb and LHb. The dMHb has been previously shown to contain a group of neurons that release the neuropeptide substance $\mathrm{P}$, and the ventral part of the $\mathrm{MHb}(\mathrm{vMHb})$ contains glutamatergic neurons $[5,38]$. According to the in situ staining of Tachykinin1 (Tac1), which acts as a precursor of substance $\mathrm{P}$, the number of substance P-ergic neurons was comparable to that in the controls (Fig. 2e, e'; m). However, Etv1, which is a member of the ETS family of transcription factors, was specifically expressed in a partition of the habenular glutamatergic neurons [33], which was significantly enlarged (Fig. $2 \mathrm{f}, \mathrm{f}^{\prime}$ ). Regarding the LHb subdivisions, we examined the LHbMC and LHbL. As visualized by the staining of Steel, the ligand for the receptor tyrosine kinase c-kit and type 2 vesicular glutamate transporter (Vglut2), the mutant Steel ${ }^{+}$LHbMC and $^{-}$ Vglut2 ${ }^{+}$LHbL expanded more broadly than the controls. However, the expression level of Steel seemed less compared with the controls (Fig. 2g-h'). Collectively, the disruption of Foxg1 led to an increased number of progenitors in the developing epithalamic VZ, which may ultimately result in defects in subtype neurons in both the MHb and LHb.

\section{Impaired habenular innervations after Foxg1 deletion}

Due to the remarkable structural alteration in the mutant epithalamus, we investigated the changes in the innervations. Immunostaining of anti-L1, which is a neural cell adhesion molecule, was performed. As shown in Fig. 3a-a', the stria medullaris (SM), which project forebrain inputs to the habenula [39], were dramatically impaired, which may also be a consequence of the severely impaired telencephalon in the mutants. The habenular commissure, which conveys information between the paired habenulas, was much thinner than that in the controls, although it could cross the midline (Fig. 3b, b'). The control processes were well fasciculated and projected dorsally across the midline. However, the mutant processes were poorly fasciculated and significantly decreased. The decrease was consistent throughout the rostro-caudal axis when observed in serial 
L1

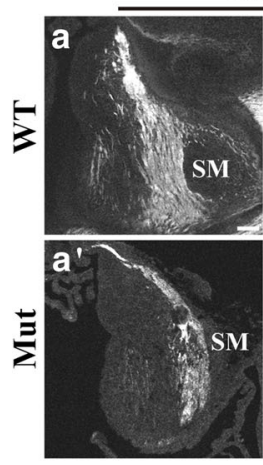

Ttr

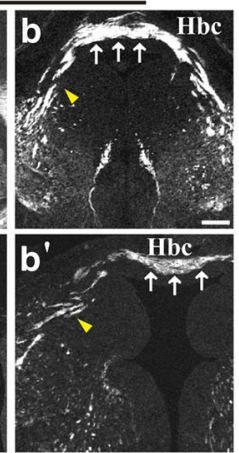

Pax6
C

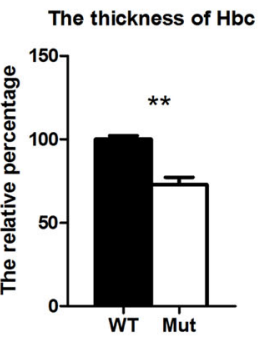

L1
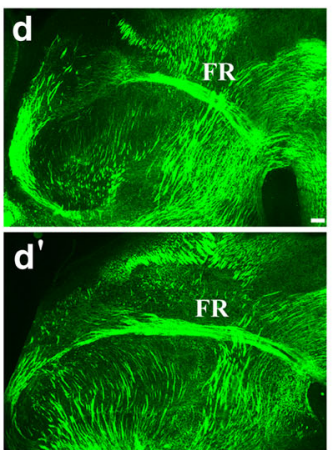

Fstl1

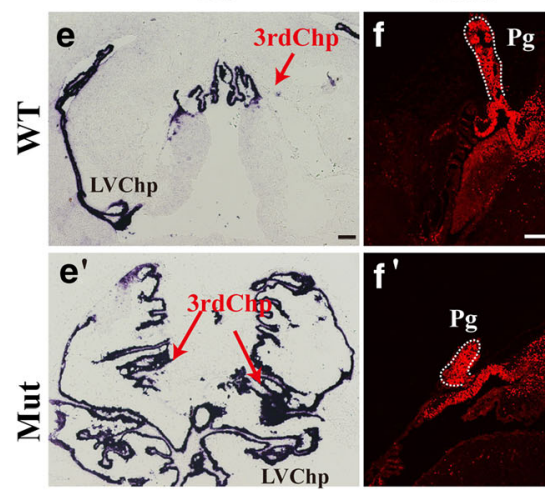

Otx2

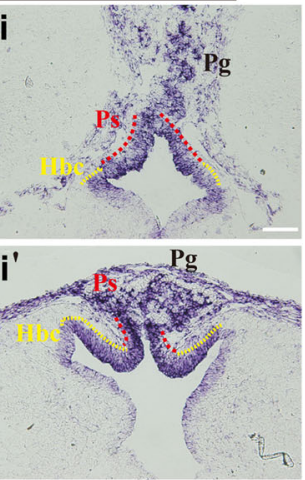

Fig. 3 Disrupted habenular innervations and abnormal diencephalic Chp and pineal gland. (a-b'): Immunofluorescence of L1 showing a reduced SM and habenular commissure (arrows in $\mathbf{b}$ and $\mathrm{b}^{\prime}$ ). Arrowhead indicates poorly fasciculated projections in the mutants compared with those in the controls. (c): Quantitative analysis of thickness of the habenular commissure at the midline area $\left(n=4,{ }^{* *} p=0.0015\right)$. (d, d'): less fasciculated FR and slightly changed projection angle. (e, e'): In situ hybridization of Ttr showing a more branched choroid plexus in the mutants. (f- $\left.-^{\prime}\right)$ : Immunofluorescence with Pax6 $\left(\mathbf{f}, f^{\prime}\right)$ and in situ hybridisation of Otx2 $\left(\mathbf{g}, g^{\prime}\right)$ and $F s t / 7\left(\mathbf{h}-\mathbf{i}^{\prime}\right)$ revealing an aberrant pineal gland, a shortened pineal stalk, which links the pineal gland to the habenula (i, i', red broken line), and a lengthened Hbc area (h, h', bracket; i, i',

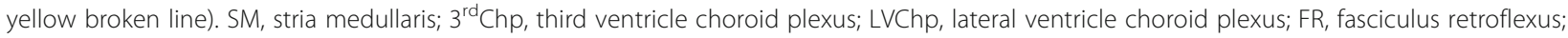
$\mathrm{Hb}$, habenula; Hbc, habenular commissure; IPN, interpeduncular nucleus; Pg, pineal gland; Ps, pineal stalk. Scale bars: $100 \mu \mathrm{m}$ (scale bar in $\mathbf{g}$ and $\left.g^{\prime}: 400 \mu \mathrm{m}\right)$

sections. We then analysed the thickness of habenular commissure in the midline area and found it was reduced by approximate $28 \%$ in Foxg1 mutants (Fig. 3c). The dramatically decreased habenular commissure was also detected by immunostaining with anti-CR and Vglut2 at E18.5 as shown in Fig. 2b, b'; h, and h'. Despite the increased numbers of neuron subtypes, the severely decreased habenular commissure indicates that neuronal differentiation may be affected by the Foxg1 deletion as well.

Interestingly, the fasciculus retroflexus (FR), through which the habenula projects to the interpeduncular nucleus (IPN) of the midbrain [39, 40], appeared to project correctly, although it was less fasciculated and its projecting angle was slightly changed, which may be due to the irregular morphology of the habenula (Fig. 3d, d'). Thus, Foxg1 is essential for the development of habenular innervations, particularly the habenular commissure.

\section{Reduced pineal gland and extremely complicated Chp} after Foxg1 deletion

During development, both the pineal gland and the $3{ }^{\text {rd }} \mathrm{Chp}$, along with the habenula, arise from the dorsal region of $\mathrm{p} 2$, which is the presumptive epithalamic domain. In addition to the habenula, the pineal gland and $3{ }^{\text {rd }}$ Chp were also impaired in Foxg1 null mutants. According to the in situ hybridization of Ttr, the $3^{\text {rd }} \mathrm{Chp}$ and lateral ventricle Chp displayed extremely complicated morphologies compared to those in the controls (Fig. 3e, e') and, to a certain extent, reflected the Chp cyst in FOXG1 patients. Meanwhile, the size of the pineal gland was reduced viewed by immunostaining of Pax6 (Fig. 3f, f'). Previous studies have shown that misregulation of Foxg1 in chick prosencephalon causes the downregulation of $O t x 2$, which is required for the development of the pineal gland and Chp [30, 41, 42]. However, here, we found that in the absence of Foxg1, the 
expression level of Otx2 in the pineal gland appears normal (Fig. 3g, g'), indicating that Otx2 is not involved in the Foxg1-regulated development of the pineal gland in mice. We observed strong expression of Follistatin-like 1 (Fstl1), which is a secreted glycoprotein that functions as an antagonist of BMP signalling in the developing pineal gland $[43,44]$. As shown in Fig. 3h-i', at E18.5 in the controls, Fstl1 was densely expressed in a distinct cell population in the pineal gland, pineal stalk, and habenula commissure. However, in the mutants, the Fstl $1^{+}$cells were not well organized, and the Fstl $1^{+}$pineal stalk was remarkably shortened with a lengthened habenula commissure. In summary, Foxg1 may be essential for the regional identities of the dorsal part of $\mathrm{p} 2$.

Early sub-regionalization of the presumptive epithalamic domain was disrupted after Foxg1 deletion

To further examine whether the regionalization of the epithalamic domain was affected by the loss of Foxg1, in situ hybridization was performed during the early developmental stage at E12.5. As shown in Fig. 4a-a", the high-level expression of the homeodomain gene $D b x 1$ normally demarcates the habenular progenitor region [45]. In the absence of Foxg1, the $\mathrm{Dbx}^{+}$domain was obviously expanded and shifted dorsolaterally (Fig. 4b-b"). The whole-mount in situ hybridization further confirmed the expansion of $D b x 1$ in Foxg1 mutants (Fig. 4a"' and b"'). Ngn2, which is a member of the proneural bHLH transcription factor family, has been shown to be widely expressed in the habenular VZ, caudal progenitor domain of the thalamus (pTH-C), and key diencephalic organizer zona limitans intrathalamica (ZLI) which is located at the interval between $\mathrm{p} 2$ and $\mathrm{p} 3$, but specifically excluded from the rostral progenitor domain of the thalamus (pTH-R) in controls [46] (Fig. 4c-c"). In the mutants, the $\mathrm{Ngn}^{+}$habenular $\mathrm{VZ}$ was expanded, while ZLI, pTH-C and pTH-R appeared comparable to those in the controls (Fig. 4d-d"). The similar results were obtained by the whole-mount hybridization (Fig. 4c"' and d"'). Next, we examined whether the primordium of the pineal gland was affected by the in situ staining of Fzd10 and found that the mutant pineal recess was
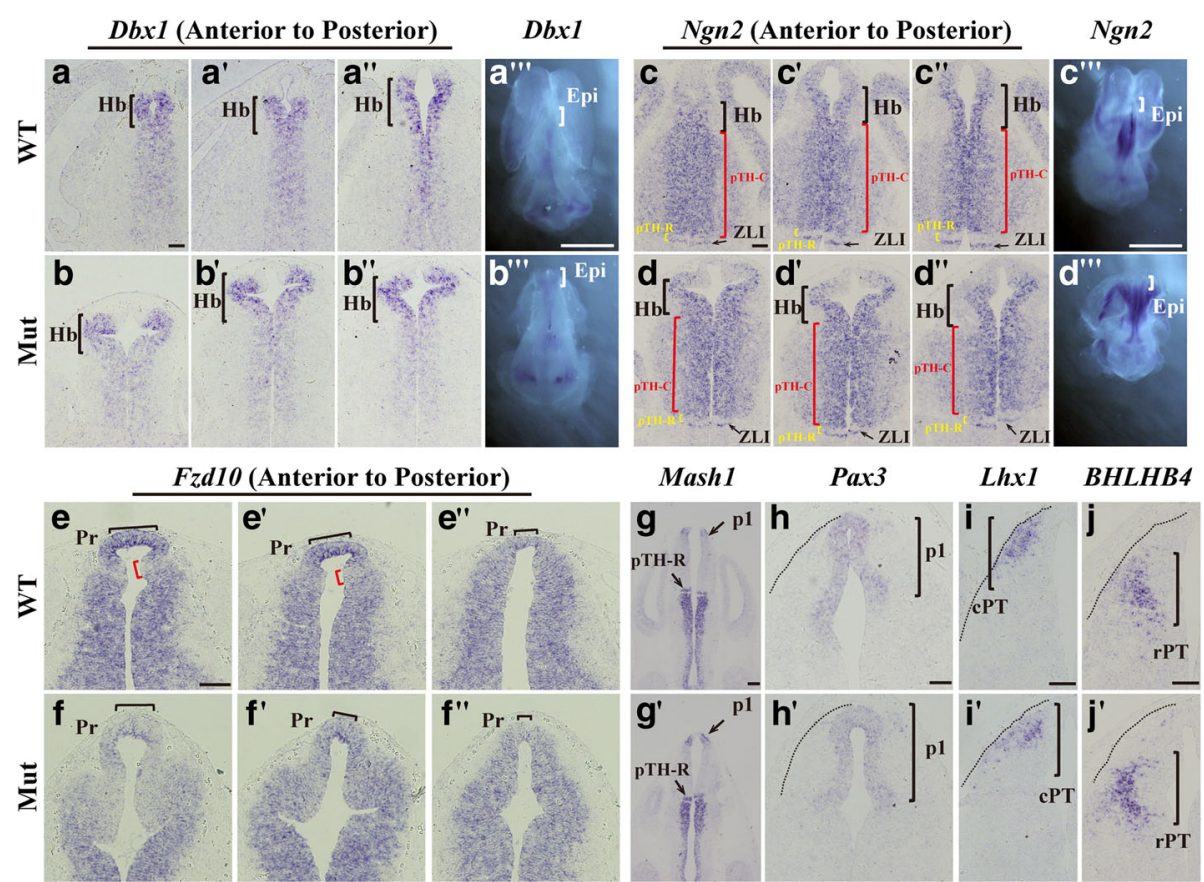

Fig. 4 Foxg 1 is required for early epithalamic sub-regionalization. (a-b"): In situ hybridization of Dbx1 showing that the habenular progenitor region (bracket) was obviously expanded and shifted dorsolaterally after Foxg1 deletion. ( $\left.\mathbf{a}^{\prime \prime \prime}, \mathbf{b}^{\prime \prime \prime}\right)$ Whole-mount in situ hybridization for Dbx 1 at E12.5 The bracket indicates the epithalamus. (c-d"): Expanded Ngn2 ${ }^{+}$habenular VZ (black bracket) but normal pTH-C (red bracket), pTH-R (yellow

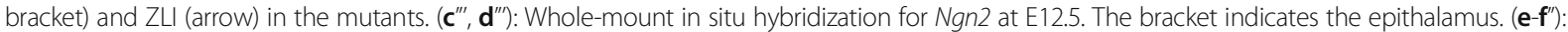
Smaller pineal recess (black bracket) shown by in situ staining of Fzd10. The red bracket marked the Fzd10 weak strip between the pineal recess and the habenular ventricle zone. (g-g): In situ hybridization of Mash1 showing normal pTH-R (arrow) and ZLI. (h-j'): No obvious changes in the patterning of p1 were revealed by the in situ staining with Pax3, which labels the pretectal VZ ( $\left.\mathbf{h}, h^{\prime}\right)$; Lhx1, which labels the mantle zone of the caudal pretectum $(\mathbf{i}, \mathbf{i})$; and BHLHB4, which labels the mantle zone of the rostral pretectum $\left(\mathbf{j}, \mathbf{j}^{\prime}\right)$. The black dashed line in $\mathbf{h}-\mathbf{j}^{\prime}$ outlined the diencephalon. Epi, epithalamus; $\mathrm{Hb}$, habenula; PTH-C, caudal progenitor domain of the thalamus; PTH-R, rostral progenitor domain of the thalamus; Pr, pineal recess; p1, prosomere 1; CPT, caudal pretectum; rPT, rostral pretectum; ZLI, zona limitans intrathalamica. Scale bars: $100 \mu \mathrm{m}$ (scale bar in $\mathbf{a}^{\prime \prime \prime}, \mathbf{b}^{\prime \prime \prime}, \mathbf{c}^{\prime \prime \prime}$ and $\mathbf{d}^{\prime \prime \prime}: 2$ mm; $\mathbf{g}$ and g': $200 \mu \mathrm{m})$ 
obviously smaller than that in the control (Fig. 4e-f"). Thus, sub-regionalization in the developing epithalamus was severely impaired.

To further investigate whether Foxg1 affects pTH-C, pTH-R and ZLI, in situ hybridization of Mash1 was also performed. As shown in Fig. 4g-g', Mash1 was strongly expressed in pTH-R and p3 but specifically excluded from the ZLI [46]. There were no differences to be detected between the control and the mutant, consistent with that viewed by in situ hybridization of $N g n 2$ (Fig. 4c-d"). We further analysed the patterning of $\mathrm{p} 1$ by Pax3, which labels the pretectal VZ [47]; LIMhomeodomain transcription factor $1(\operatorname{Lh} \times 1)$, which is a commonly used marker of the mantle zone of the caudal pretectum [48]. BHLHB4, which is a member of the basic helix-loop-helix (bHLH) family; and a specific marker for the mantle zone of the rostral pretectum [49]. No obvious alterations were observed (Fig. 4h-j'). Collectively, Foxg1 is required for the sub-regionalization of the presumptive epithalamic domain but has no effects on the other parts of p2 and p1 during early diencephalic development.

\section{Shh and Fgf signalling were not affected during the development of the epithalamus}

Multiple signals, including fibroblast growth factor (Fgfs) are expressed in the dorsal midline of the diencephalon
[15] and coordinate with Shh, which is secreted from the ZLI and basal plate, to establish regional identity in the developing diencephalon [50, 51]. Fgf8 has been reported to be involved in the patterning of the p2 region. In Fgf8 hypomorphic mice, the pineal gland and habenula are lost or reduced, exhibiting dose-dependent changes [15]. Fgf15, which is another member of the Fgf family, has been shown to act as a downstream target of Shh that regulates the development of the diencephalon [52]. Using in situ hybridization, we explored whether Fgf signalling is involved in the regulation of Foxg1 in the subregionalization of the epithalamic domain. As shown in Fig. 5a, at E12.5, Fgf15 was expressed in the future habenula and thalamic VZ with no detectable expression in the developing pineal gland and $3^{\text {rd }} \mathrm{Chp}$ in the controls; the transcription level of Fgf15 in the mutant presumptive habenula was comparable to that of controls, no significant changes were observed in p2 (Fig. 5a, a', arrowhead). The same result was obtained using wholemount hybridization and qRT-PCR (Fig. 5b, b'; c). No detectable changes were observed in Fgf8 either along the AP axis (Fig. 5d-d"; e-e"). Therefore, Fgf15, as well as Fgf8, were not involved in the Foxg1-mediated regulation of the development of the epithalamus. Previously $W n t 3 a$ has been reported to be expressed in the dorsal p2 and Wnt8b is expressed in the prethalamus [15].

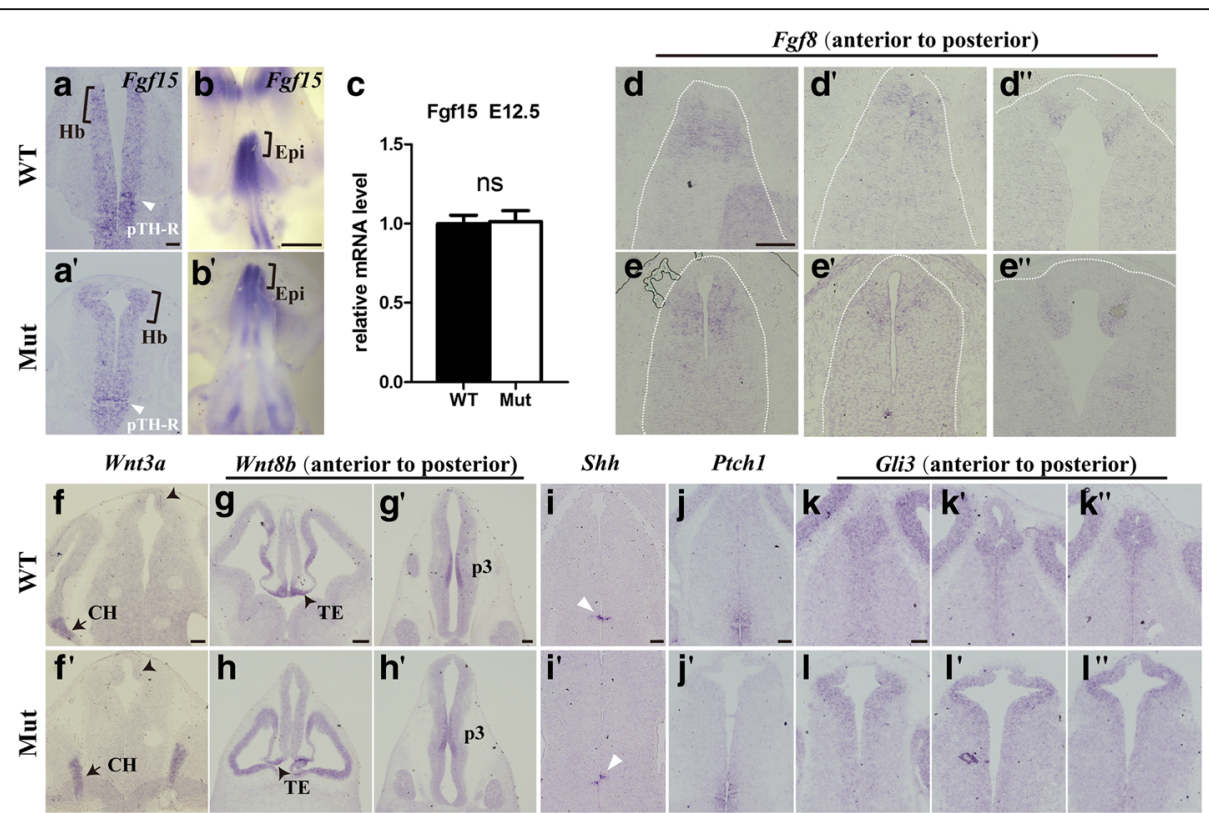

Fig. 5 Shh and Fgf signalling were not affected in the epithalamic development. (a-a'): Transcription level of Fgf15 in the presumptive habenula (bracket) was not obviously affected in the mutants, no changes were observed in the region of pTH-R (arrowhead) in p2. (b-b'): The whole mount in situ from E12.5 embryos also showing a comparable transcription level in the epithalamus (bracket). c: Relative mRNA levels of Fgf15 ( $n=4$, $p=0.898)$. (d-e'): No obvious differences in Fgf8 were observed in the Foxg1 mutants. The white dashed line outlined the diencephalon. (f-h'): Staining of Wnt3a in dorsal P2 and Wnt8b in TE and P3 in mutants were comparable to that of controls. Arrows in $\mathbf{f}$ and $\mathrm{f}^{\prime}$ indicate the cortical hem, arrowheads in $\mathbf{g}$ and $\mathbf{h}$ indicate the thalamic eminence. (i-I'): The activity of the Shh signalling pathway appeared normal in the mutants. The white arrow in $\mathbf{i}$ and $\mathbf{i}^{\prime}$ indicates the ZLI. CH, cortical hem; Epi, epithalamus; Hb, habenula; IPN, interpeduncular nucleus; TE, thalamic eminence; ZLI, zona limitans intrathalamica. Scale bars: $100 \mu \mathrm{m}$ (scale bar in b and b': 1 mm, g-h': $200 \mu \mathrm{m}$ ) 
Foxg1 has been shown to suppress Wnt function in the developing telencephalon $[15,53]$. To explore whether Foxg1 regulate the development of epithaluams through Wnt signalling, we then examined Wnt3a and Wnt8b; however, no obvious alteration were detected either (Fig. 5f-h').

Shh, which is secreted by the ZLI, is critical for the development of the diencephalon [50, 54-56]. The ventral high-dorsal ${ }^{\text {low }}$ gradient of Shh specifies the diencephalic regional identity [57]. Therefore, we examined whether Shh signalling contributes to the epithalamic defects. At E12.5, in the Foxg1 mutants, the expression of Shh in the ZLI was comparable to that in the controls (Fig. 5i, i'). We then examined the activity of the Shh signalling pathway as reflected by Ptch1 [58]. No obvious differences were detected (Fig. 5j, j'). Meanwhile, the level of Gli3, which is a member of the Glioma-associated oncogene (Gli) family that has been reported to inhibit Shh signalling [59], also appeared normal in the epithalamic VZ (Fig. 5k-k"; 1-l"). Collectively, Foxg1 may regulate the development of the epithalamus independently of Shh signalling.

\section{Discussion}

Foxg1 has been reported to be critical for telencephalic development $[17,19]$. However, its role in the development of the diencephalon remains unclear. Individuals with FOXG1 syndrome exhibit a disturbed sleep pattern, Chp cysts and emotional disorders [21-23], suggesting that Foxg1 likely plays a role in epithalamic development. In this study, we demonstrate that Foxg1 is essential for the development of the epithalamus. The disruption of Foxg1 leads to an extremely complicated Chp, a reduced pineal gland and an enlarged habenula. Moreover, we demonstrate that Foxg1 may be required for the regional specification of the epithalamic progenitor domain independently of Shh and Fgf signalling. Our findings shed light on the molecular mechanism underlying the subdivision of the epithalamic domain.

Previously, the function of Foxg1 was underinvestigated in the developing diencephalon. In this study, we identified specific expression of Foxg1 in the dorsal part of $\mathrm{p} 2$ from which the epithalamus derives and further elucidated its function during epithalamic development. The habenula has been reported to be closely related to emotional disorders and has recently attracted increasing attention $[2,9,10]$. By receiving inputs from the limbic system and basal ganglia and projecting to monoaminergic nuclei, the habenula acts as a node that connects the forebrain to the brainstem [39]. Here, we found that the disruption of Foxg1 results in an enlarged habenula with an increased number of subtype habenular neurons. The loss of Foxg1 also caused differentiation defects in habenular neurons, which led to impaired habenular innervations and ultimately resulted in abnormal information conveyance among the forebrain, brainstem and paired habenula, which may account for the emotional disorders observed in patients with FOXG1 mutations. To the best of our knowledge, this is the first report illustrating that Foxg1 regulates the development of the epithalamus.

During development, the most dorsal domain of $\mathrm{p} 2$ gives rise to the epithalamus, which consists of $3{ }^{\text {rd }} \mathrm{Chp}$, pineal gland, habenular commissure and habenula. However, the mechanism by which the sub-regional identities are established is unknown. Multiple signals, including Fgfs have been found to be specifically expressed in the dorsal region of $\mathrm{p} 2$ and involved in the development of the epithalamus $[15,60,61]$. Previous studies have shown that the pineal gland and habenula are lost or reduced in Fgf8 hypomorphic mice, which exhibit dosagedependent changes in the epithalamus [15]. Previously, we have reported a strong expression of Fstl1 in the pineal gland [43]. In this study, we have detected Fstl1 is also expressed in the habenular commissure. The remarkably shorten pineal stalk with the lengthened habenular commissure observed in the Foxg1 mutants indicate Fstl1 may be required for the development of the pineal gland and the habenular commissure. Further study is needed to elucidate its function. Shh is secreted from the ZLI and basal plate, and by coordinating with signals from the dorsal region, Shh is critical for the regionalization of the diencephalon $[45,50,51]$. Fgf15 has been identified as a downstream target of Shh that suppresses cell proliferation and promotes differentiation in the developing telencephalon [62]. In this study, we did not detect obvious changes in Shh and Fgf signalling in our mutants. The mutant ZLI was comparable to that in the controls, and the activity of the Shh signalling pathway, as shown by Ptch1 and Gli3, appeared normal, suggesting that the regulation of Foxg1 during epithalamic development is independent of Shh and Fgf signallings. Further studies are required to determine the downstream targets of Foxg1 during the development of the epithalamus.

\section{Conclusions}

In the present study, we have identified a specific expression of Foxg1 in the developing epithalamus and further found that disruption of Foxg1 resulted in an impaired epithalamus with an expanded habenula, a reduced pineal gland and more branched choroid plexus. No obvious changes in Shh and Fgf signaling were detected in Foxg1 mutants, indicating that Foxg1 may regulates the development of the epithalamus independent of Shh and Fgfs. Our findings provide new insights into the regulation of the development of the epithalamus. Further study is required to elucidate the molecular mechanism. 


\section{Abbreviations}

3rdChp: Third ventricle choroid plexus; $3^{\text {rd }} \mathrm{V}$ : Third ventricle; $3^{\text {rd }} \mathrm{VZ}$ : Ventricular zone of the third ventricle; $\mathrm{CH}$ : Cortical hem; CPT: Caudal pretectum; dMHb: Dorsal medial habenula; FR: Fasciculus retroflexus; $\mathrm{Hb}$ : Habenula; Hbc: Habenular commissure; IPN: Interpeduncular nucleus; LHb: Lateral habenula; LHbL: Lateral division of the lateral habenula; LHbMC: Central part of the medial division of the lateral habenula; MHb: Medial habenula; p: Prosomere; Pg: Pineal gland; Pr: Pineal recess; Ps: Pineal stalk; PTH-C: Caudal progenitor domain of the thalamus; PTH-R: Rostral progenitor domain of the thalamus; rPT: Rostral pretectum; SM: Stria medullaris; TE: Thalamic eminence; vMHb: Ventral medial habenula; ZLI: Zona limitans intrathalamica

\section{Acknowledgements}

We would like to thank Mr. Yiquan Wei and Ms. Li Liu for their assistance with the laboratory and animal care, Xiaochun Gu and other members of the laboratory for discussions.

\section{Ethical approval and consent to participate}

Not applicable.

\section{Funding}

This study was supported by grant 2016YFA0501001 from the Ministry of Science and Technology of China and grants 91232301 and 31471041 from the National Natural Science Foundation of China to C.Z. and 31500844 from the National Natural Science Foundation of China to X.W.

\section{Availability of data and materials}

The generated data are included in this published article. The mouse lines and antiprobes are available from the corresponding author upon request.

\section{Authors' contributions}

Designed the study: BL and CZ. Conducted the experiments: BL. Analysed and interpreted the data: $\mathrm{BL}, \mathrm{CZ}, \mathrm{XW}$ and $\mathrm{KZ}$. Prepared the manuscript: $\mathrm{BL}$ and $\mathrm{CZ}$. All authors read and approved the final manuscript.

\section{Consent for publication}

Not applicable.

\section{Competing interests}

The authors declare that they have no competing interests.

\section{Publisher's Note}

Springer Nature remains neutral with regard to jurisdictional claims in published maps and institutional affiliations.

Received: 19 October 2017 Accepted: 24 January 2018

Published online: 02 February 2018

\section{References}

1. Andres $\mathrm{KH}$, von During $\mathrm{M}$, Veh RW. Subnuclear organization of the rat habenular complexes. J Comp Neurol. 1999;407(1):130-50.

2. Lecourtier L, Neijt HC, Kelly PH. Habenula lesions cause impaired cognitive performance in rats: implications for schizophrenia. Eur J Neurosci. 2004;19(9):2551-60.

3. Mathuru AS, Jesuthasan $\mathrm{S}$. The medial habenula as a regulator of anxiety in adult zebrafish. Front Neural circuits. 2013;7:99.

4. Klemm WR. Habenular and interpeduncularis nuclei: shared components in multiple-function networks. Med Sci Monit. 2004:10(11):RA261-73.

5. Lecourtier L, Kelly PH. A conductor hidden in the orchestra? Role of the habenular complex in monoamine transmission and cognition. Neurosci Biobehav Rev. 2007;31(5):658-72.

6. Klein DC, Bailey MJ, Carter DA, Kim JS, Shi Q, Ho AK, Chik CL, Gaildrat P, Morin F, Ganguly $S$, et al. Pineal function: impact of microarray analysis. Mol Cell Endocrinol. 2010;314(2):170-83.

7. Lehtinen MK, Zappaterra MW, Chen X, Yang YJ, Hill AD, Lun M, Maynard T, Gonzalez D, Kim S, Ye P, et al. The cerebrospinal fluid provides a proliferative niche for neural progenitor cells. Neuron. 2011;69(5):893-905.

8. Currle DS, Cheng X, Hsu CM, Monuki ES. Direct and indirect roles of CNS dorsal midline cells in choroid plexus epithelia formation. Development. 2005;132(15):3549-59.
9. Li B, Piriz J, Mirrione M, Chung C, Proulx CD, Schulz D, Henn F, Malinow R. Synaptic potentiation onto habenula neurons in the learned helplessness model of depression. Nature. 2011;470(7335):535-9.

10. Li K, Zhou T, Liao L, Yang Z, Wong C, Henn F, Malinow R, Yates JR 3rd, $\mathrm{Hu} \mathrm{H}$. betaCaMKII in lateral habenula mediates core symptoms of depression. Science. 2013;341(6149):1016-20.

11. Valjakka A, Vartiainen J, Tuomisto L, Tuomisto JT, Olkkonen H, Airaksinen MM. The fasciculus retroflexus controls the integrity of REM sleep by supporting the generation of hippocampal theta rhythm and rapid eye movements in rats. Brain Res Bull. 1998:47(2):171-84.

12. Wu W, Cui L, Fu Y, Tian Q, Liu L, Zhang X, Du N, Chen Y, Qiu Z, Song Y, et al. Sleep and cognitive abnormalities in acute minor thalamic infarction. Neurosci Bull. 2016:32(4):341-8.

13. Puelles $L$, Rubenstein $J$. Expression patterns of homeobox and other putative regulatory genes in the embryonic mouse forebrain suggest a neuromeric organization. Trends Neurosci. 1993;16(11):472-9.

14. Puelles $L$, Rubenstein JL. Forebrain gene expression domains and the evolving prosomeric model. Trends Neurosci. 2003;26(9):469-76.

15. Martinez-Ferre A, Martinez S. The development of the thalamic motor learning area is regulated by Fgf8 expression. J Neurosci. 2009;29(42):13389-400.

16. Clanton JA, Hope KD, Gamse JT. Fgf signaling governs cell fate in the zebrafish pineal complex. Development. 2012;140(2):323-32.

17. Xuan S, Baptista CA, Balas G, Tao W, Soares VC, Lai E. Winged helix transcription factor BF-1 is essential for the development of the cerebral hemispheres. Neuron. 1995;14(6):1141-52.

18. Pratt T, Quinn JC, Simpson TI, West JD, Mason JO, Price DJ. Disruption of early events in thalamocortical tract formation in mice lacking the transcription factors Pax6 or Foxg1. J Neurosci. 2002;22(19):8523-31.

19. Tian C, Gong Y, Yang Y, Shen W, Wang K, Liu J, Xu B, Zhao J, Zhao C. Foxg1 has an essential role in postnatal development of the dentate gyrus. J Neurosci. 2012;32(9):2931-49.

20. Yang Y, Shen W, Ni Y, Su Y, Yang Z, Zhao C. Impaired interneuron development after Foxg1 disruption. Cereb Cortex. 2017;27(1):793-808.

21. Kortum F, Das S, Flindt M, Morris-Rosendahl DJ, Stefanova I, Goldstein A Horn D, Klopocki E, Kluger G, Martin P, et al. The core FOXG1 syndrome phenotype consists of postnatal microcephaly, severe mental retardation, absent language, dyskinesia, and corpus callosum hypogenesis. J Med Genet. 2011:48(6):396-406

22. Brunetti-Pierri N, Paciorkowski AR, Ciccone R, Della Mina E, Bonaglia MC, Borgatti R, Schaaf CP, Sutton VR, Xia Z, Jelluma N, et al. Duplications of FOXG1 in 14q12 are associated with developmental epilepsy, mental retardation, and severe speech impairment. Eur J Human Genet. 2011;19(1):102-7.

23. Allou L, Lambert L, Amsallem D, Bieth E, Edery P, Destree A, Rivier $F$, Amor D, Thompson E, Nicholl J, et al. 14q12 and severe Rett-like phenotypes: new clinical insights and physical mapping of FOXG1-regulatory elements. Eur J Human Genet. 2012;20(12):1216-23.

24. Hebert JM, McConnell SK. Targeting of cre to the Foxg1 (BF-1) locus mediates loxP recombination in the telencephalon and other developing head structures. Dev Biol. 2000;222(2):296-306.

25. Gu X, Yan Y, Li H, He D, Pleasure SJ, Zhao C. Characterization of the Frizzled10-CreER transgenic mouse: an inducible Cre line for the study of Cajal-Retzius cell development. Genesis. 2009;47(3):210-6.

26. Zhao C, Guan W, Pleasure SJ. A transgenic marker mouse line labels Cajal-Retzius cells from the cortical hem and thalamocortical axons. Brain Res. 2006;1077(1):48-53.

27. Correia KM, Conlon RA. Whole-mount in situ hybridization to mouse embryos. Methods. 2001;23(4):335-8.

28. Ansorg A, Witte OW, Urbach A. Age-dependent kinetics of dentate gyrus neurogenesis in the absence of cyclin D2. BMC Neurosci. 2012;13:46.

29. Noguchi H, Murao N, Kimura A, Matsuda T, Namihira M, Nakashima K. DNA Methyltransferase 1 is indispensable for development of the Hippocampal dentate Gyrus. J Neurosci. 2016;36(22):6050-68.

30. Aguiar DP, Sghari S, Creuzet S. The facial neural crest controls fore- and midbrain patterning by regulating Foxg 1 expression through Smad1 activity. Development. 2014;141(12):2494-505.

31. Kawaguchi D, Sahara S, Zembrzycki A, O'Leary DDM. Generation and analysis of an improved Foxg1-IRES-Cre driver mouse line. Dev Biol. 2016;412(1):139-47.

32. Li K, Zhang J, Li JY. Gbx2 plays an essential but transient role in the formation of thalamic nuclei. PLoS One. 2012;7(10):e47111. 
33. Quina LA, Wang S, Ng L, Turner EE. Brn3a and Nurr1 mediate a gene regulatory pathway for habenula development. J Neurosci. 2009;29(45):14309-22.

34. Rash BG, Grove EA. Shh and Gli3 regulate formation of the telencephalicdiencephalic junction and suppress an isthmus-like signaling source in the forebrain. Dev Biol. 2011;359(2):242-50.

35. Bulchand S, Grove EA, Porter FD, Tole S. LIM-homeodomain gene Lhx2 regulates the formation of the cortical hem. Mech Dev. 2001;100(2):165-75.

36. Yan Y, Li Y, Hu C, Gu X, Liu J, Hu YA, Yang Y, Wei Y, Zhao C. Expression of Frizzled10 in mouse central nervous system. Gene Expr Patterns. 2009;9(3):173-7

37. Frassoni $C$, Arcelli $P$, Selvaggio M, Spreafico R. Calretinin immunoreactivity in the developing thalamus of the rat: a marker of early generated thalamic cells. Neuroscience. 1998;83(4):1203-14.

38. Contestabile A, Villani L, Fasolo A, Franzoni MF, Gribaudo L, Oktedalen O, Fonnum F. Topography of cholinergic and substance $P$ pathways in the habenulo-interpeduncular system of the rat. An immunocytochemical and microchemical approach. Neuroscience. 1987;21(1):253-70.

39. Bianco $\mathrm{H}$, Wilson SW. The habenular nuclei: a conserved asymmetric relay station in the vertebrate brain. Philos Trans R Soc Lond Ser B Biol Sci. 2009:364(1519):1005-20.

40. Contestabile A, Flumerfelt BA. Afferent connections of the interpeduncular nucleus and the topographic organization of the habenulo-interpeduncular pathway: an HRP study in the rat. J Comp Neurol. 1981;196(2):253-70.

41. Nishida A, Furukawa A, Koike C, Tano Y, Aizawa S, Matsuo I, Furukawa T. Otx2 homeobox gene controls retinal photoreceptor cell fate and pineal gland development. Nat Neurosci. 2003;6(12):1255-63.

42. Johansson PA, Irmler M, Acampora D, Beckers J, Simeone A, Gotz M. The transcription factor Otx2 regulates choroid plexus development and function. Development. 2013;140(5):1055-66.

43. Yang Y, Liu J, Mao H, Hu YA, Yan Y, Zhao C. The expression pattern of Follistatin-like 1 in mouse central nervous system development. Gene Expr Patterns. 2009;9(7):532-40.

44. Geng Y, Dong Y, Yu M, Zhang L, Yan X, Sun J, Qiao L, Geng H, Nakajima M, Furuichi T, et al. Follistatin-like 1 (Fstl1) is a bone morphogenetic protein (BMP) 4 signaling antagonist in controlling mouse lung development. Proc Natl Acad Sci U S A. 2011;108(17):7058-63.

45. Chatterjee M, Guo Q, Weber S, Scholpp S, Li JY. Pax6 regulates the formation of the habenular nuclei by controlling the temporospatial expression of Shh in the diencephalon in vertebrates. BMC Biol. 2014;12(1):13

46. Vue TY, Aaker J, Taniguchi A, Kazemzadeh C, Skidmore JM, Martin DM, Martin JF, Treier M, Nakagawa Y. Characterization of progenitor domains in the developing mouse thalamus. J Comp Neurol. 2007;505(1):73-91.

47. Ferran JL, Sanchez-Arrones L, Bardet SM, Sandoval JE, Martinez-de-la-Torre M, Puelles L. Early pretectal gene expression pattern shows a conserved anteroposterior tripartition in mouse and chicken. Brain Res Bull. 2008;75(2-4):295-8.

48. Suda Y, Hossain ZM, Kobayashi C, Hatano O, Yoshida M, Matsuo I, Aizawa S. Em×2 directs the development of diencephalon in cooperation with Otx2. Development. 2001;128(13):2433-50.

49. Bramblett DE, Copeland NG, Jenkins NA, Tsai MJ. BHLHB4 is a bHLH transcriptional regulator in pancreas and brain that marks the dimesencephalic boundary. Genomics. 2002;79(3):402-12.

50. Martinez-Ferre A, Martinez S. Molecular regionalization of the diencephalon. Front Neurosci. 2012;6:73.

51. Jeong Y, Dolson DK, Waclaw RR, Matise MP, Sussel L, Campbell K, Kaestner KH, Epstein DJ. Spatial and temporal requirements for sonic hedgehog in the regulation of thalamic interneuron identity. Development. 2011;138(3):531-41.

52. Martinez-Ferre A, Lloret-Quesada C, Prakash N, Wurst W, Rubenstein JL, Martinez S. Fgf15 regulates thalamic development by controlling the expression of proneural genes. Brain Struct Funct. 2015;221(6):3095-109.

53. Danesin C, Peres JN, Johansson M, Snowden V, Cording A, Papalopulu N, Houart C. Integration of telencephalic Wnt and hedgehog signaling center activities by Foxg1. Dev Cell. 2009;16(4):576-87.

54. Hashimoto-Torii K, Motoyama J, Hui CC, Kuroiwa A, Nakafuku M, Shimamura K. Differential activities of sonic hedgehog mediated by Gli transcription factors define distinct neuronal subtypes in the dorsal thalamus. Mech Dev. 2003;120(10):1097-111.

55. Kiecker $\mathrm{C}$, Lumsden $\mathrm{A}$. Hedgehog signaling from the $\mathrm{ZLI}$ regulates diencephalic regional identity. Nat Neurosci. 2004;7(11):1242-9.
56. Scholpp S, Wolf O, Brand M, Lumsden A. Hedgehog signalling from the zona limitans intrathalamica orchestrates patterning of the zebrafish diencephalon. Development. 2006;133(5):855-64.

57. Vue TY, Bluske K, Alishahi A, Yang LL, Koyano-Nakagawa N, Novitch B, Nakagawa $Y$. Sonic hedgehog signaling controls thalamic progenitor identity and nuclei specification in mice. J Neurosci. 2009;29(14):4484-97.

58. Agren M, Kogerman P, Kleman MI, Wessling M, Toftgard R. Expression of the PTCH1 tumor suppressor gene is regulated by alternative promoters and a single functional Gli-binding site. Gene. 2004;330:101-14.

59. Persson M, Stamataki D, te Welscher P, Andersson E, Bose J, Ruther U, Ericson J, Briscoe J. Dorsal-ventral patterning of the spinal cord requires Gli3 transcriptional repressor activity. Genes Dev. 2002;16(22):2865-78.

60. Lim Y, Cho G, Minarcik J, Golden J. Altered BMP signaling disrupts chick diencephalic development. Mech Dev. 2005;122(4):603-20.

61. Louvi A, Yoshida M, Grove EA. The derivatives of the Wnt3a lineage in the central nervous system. J Comp Neurol. 2007;504(5):550-69.

62. Borello U, Cobos I, Long JE, McWhirter JR, Murre C, Rubenstein JL. FGF15 promotes neurogenesis and opposes FGF8 function during neocortical development. Neural Dev. 2008;3:17.

\section{Submit your next manuscript to BioMed Central and we will help you at every step:}

- We accept pre-submission inquiries

- Our selector tool helps you to find the most relevant journal

- We provide round the clock customer support

- Convenient online submission

- Thorough peer review

- Inclusion in PubMed and all major indexing services

- Maximum visibility for your research

Submit your manuscript at www.biomedcentral.com/submit
Biomed Central 